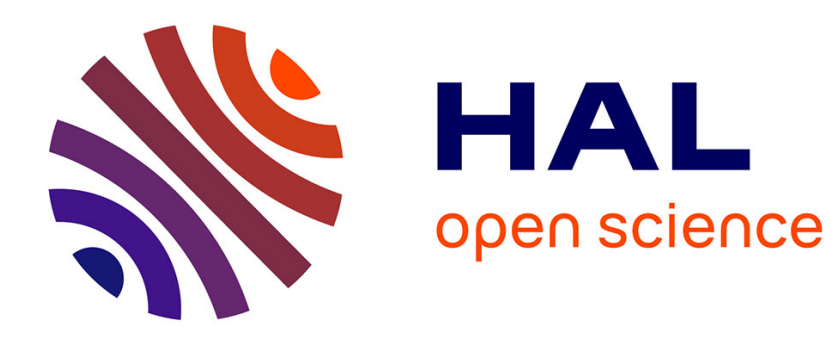

\title{
Sur le ferromagnétisme des ferrites, ou ferrimagnétisme
} Louis Néel

\section{To cite this version:}

Louis Néel. Sur le ferromagnétisme des ferrites, ou ferrimagnétisme. Physica, 1950, 16 (3), pp.350-352. 10.1016/0031-8914(50)90034-6 . hal-02888382

\section{HAL Id: hal-02888382 \\ https://hal.science/hal-02888382}

Submitted on 29 Jul 2020

HAL is a multi-disciplinary open access archive for the deposit and dissemination of scientific research documents, whether they are published or not. The documents may come from teaching and research institutions in France or abroad, or from public or private research centers.
L'archive ouverte pluridisciplinaire HAL, est destinée au dépôt et à la diffusion de documents scientifiques de niveau recherche, publiés ou non, émanant des établissements d'enseignement et de recherche français ou étrangers, des laboratoires publics ou privés. 


\section{LETTRE A LA REDACTION}

\section{Sur le ferromagnétisme des ferrites, ou ferrimagnétisme}

Les travaux de A. Serre ${ }^{1}$ ) et surtout ceux de J. L. S n o e k ${ }^{2}$ ) ont attiré l'attention sur les remarquables propriétés ferromagnétiques des ferrites à structure de spinelle. Dans ces corps, les ions métalliques occupent soit les sites tétraèdriques, ou sites $A$, entourés de 4 atomes d'oxygène, soit les sites octaèdriques, ou sites $B$, entourés de 6 atomes d'oxygène: on compte 1 site $A$ et 2 sites $B$ par molécule $\mathrm{Fe}_{2} \mathrm{O}_{3} \mathrm{MO}$. Dans les ferrites normaux, les ions $\mathrm{Fe}^{+++}$sont en $B$ et l'ion $\mathrm{M}^{++}$en $A$. B a r $\mathrm{th}$ et $\mathrm{P}$ os n ja k ${ }^{3}$ ) ont montré qu'il existait aussi des ferrites inverses dans lesquels un ion $\mathrm{Fe}^{+++}$ est en $A$, tandis que les deux autres ions $\mathrm{Fe}^{+++}$et $\mathrm{M}^{++}$sont en $B$. V e rwe y et $\mathrm{H}$ eil $\mathrm{mann}{ }^{4}$ ) ont prouvé ques les ferrites ferromagnétiques sont inverses tandis que les ferrites de zinc et de cadmium, paramagnétiques, sont normaux.

Récemment, J. H. Gis o $1 \mathrm{f}^{5}$ ) a proposé une interprétation selon laquelle seuls les ions métalliques sur les sites $A$, c'est-à-dire les ions $\mathrm{Fe}^{+++}$, contribueraient au ferromagnétisme. Cette hypothèse soulève quelques difficultés. En premier lieu, elle donne un moment moléculaire à saturation $M_{s}$ égal au moment de l'ion $\mathrm{Fe}^{+++}$, soit $5 \mu_{B}$ ( $\mu_{B}=$ magnéton de $\left.\mathrm{B} \circ \mathrm{h} \mathrm{r}\right)$, tandis que de nombreuses expériences indépendantes ( $\mathrm{E}$. W. G o r t e $\mathrm{r}^{6}$ ) à Eindhoven, C. Guilla $\mathrm{ud}^{7}$ ) à Bellevue, L. Weil, L. Bochirol et $\mathrm{R}$. $\mathrm{P}$ a u t h e $\mathrm{n} \mathrm{e} \mathrm{t}{ }^{8}$ ) à Grenoble) ont respectivement donné $5,00 \mu_{B}$, $4,06 \mu_{B}, 3,35 \mu_{B}, 2,25, \mu_{B}, 1,37 \mu_{B}$ en choisissant successivement $\mathrm{Mn}, \mathrm{Fe}$, $\mathrm{Co}, \mathrm{Ni}, \mathrm{Cu}$ pour jouer le rôle de l'ion $\mathrm{M}^{++}$. Ces valeurs montreraient plutôt que c'est l'ion M qui occupe le site $A$ mais alors ces ferrites ne seraient pas inverses mais normaux, en contradiction avec les résultats de $\mathrm{V}$ e $\mathrm{r}$ we y. En second lieu, l'hypothèse de $\mathrm{G}$ i s ol $\mathrm{f}$, appliquée aux ferrites mixtes $\mathrm{Fe}_{2} \mathrm{O}_{3}$, xMO, $(1-x) \mathrm{ZnO}$, indique que $M_{s}$ décroît linéairement de 5 à $0 \mu_{B}$ lorsque $x$ croît de 0 à 1 , tandis que E. W. Gor te r (loc. cit.) a montré que pour $\mathrm{M}=\mathrm{Mn}, \mathrm{Fe}, \mathrm{Co}, \mathrm{Ni}, \mathrm{Cu}, \mathrm{Li}_{0.5}+\mathrm{Fe}_{0.5}^{++}+$, le moment commence par croître lorsque $x$ diminue, la tangente initiale à la courbe $M_{s}=f(x)$ s'extrapolant vers $10 \mu_{B}$ pour $x=0$. En troisième lieu, dans le ferrite de cuivre, qui est un ferrite inverse assez proche de la limite des ferrites normaux, les ions métalliques tendent à haute température à se répartir statistiquement sur les sites $A$ et les sites $B$ : cet état peut être partiellement conservé par trempe. Si $M_{s}$ provient des ions situés en $A$, il doit être à l'état trempé plus petit qu'à l'état recuit puisque le nombre des ions $\mathrm{Fe}^{+++}$y est moindre: les expériences de E. W. Gorter indiquent le contraire. Enfin, dans la même hypothèse, la variation thermique de la susceptibilité magnétique $\chi$ 
au dessus du point de $\mathrm{C}$ u ri e $\theta_{f}$ devrait être analogue à celle des autres ferromagnétiques, soit en gros une variation linéaire de $1: \chi$ en fonction de la température $T$, avec au voisinage de $\theta$, une convexité vers l'axe des $T$. L'expérience donne au contraire une courbe d'allure hyperbolique avec sa concavité vers l'axe des $T$. L'interprétation de G is olf ne paraît donc pas admissible.

J'avais antérieurement proposé une autre hypothèse ${ }^{9}$ ) selon laquelle les interactions d'échange prépondérantes sont essentiellement des interactions négatives entre les ions placés sur les sites $A$ et les ions placés sur les sites $B$. Il en résulte qu'à basse température les moments des ions situés en $A$ s'alignent parallèlement entre eux pour donner une résultante $M_{a}$ et que les moments des ions situés en $B$ s'alignent en sens inverse avec une résultante $M_{b}$ : le moment résultant observable $M_{s}$ est égal à la différence $M_{b}-M_{a}$. Ainsi, les moments des deux ions $\mathrm{Fe}^{+++}$d'un ferrite inverse s'annulent puisqu'ils sont situés, l'un en $A$ et l'autre en $B$. Le moment $M_{s}$ est donc simplement égal à celui de l'ion $\mathrm{N}^{++}$. On rend alors compte quantitativement des valeurs de $M_{s}$ pour les ferrites de $\mathrm{Mn}, \mathrm{Fe}, \mathrm{Co}, \mathrm{Ni}, \mathrm{Cu}, \mathrm{Li}$. On interprète aussi les variations de $M_{s}$ avec la concentration dans les ferrites mixtes $\mathrm{M}-\mathrm{Zn}$ ainsi que l'extrapolation vers $10 \mu_{B}$ de la tangente initiale à la courbe $M_{s}=f(x)$ : en effet, le remplacement d'un ion $M$ de moment $m$, placé en $B$, par un ion $Z_{n}$ de moment nul, placé en $A$, provoque une variation de moment égale à $(10-m) \mu_{B}$, le premier terme provenant du retournement du moment de 'lion $\mathrm{Fe}^{+++}$qui passe de $A$ en $B$, le second du départ de l'ion $M$. L'augmentation du moment du ferrite de cuivre par la trempe s'explique aussi aisément. Enfin, l'extension de la théorie du champ moléculaire de W e is s permet de montrer que la susceptibilité au dessus du point de $\mathrm{C}$ u $\mathrm{r}$ i e obéit à la relation

$$
\frac{1}{\chi}=\frac{T}{C}+\frac{1}{\chi_{0}}-\frac{\sigma}{T-\theta} .
$$

où $C$ est la constante de $C \mathrm{ur}$ i e relative à tous les ions présents et $\chi_{0}, \sigma, \theta$ sont trois coefficients calculables en fonction des trois coefficients de champ moléculaire $n_{a a}, n_{a b}, n_{b b}$ qui définissent les interactions. Effectivement, on représente ainsi convenablement les résultats expérimentaux et on en déduit $n_{a a}, n_{a b}$ et $n_{b b}$. Ces coefficients une fois connus, il est alors possible de calculer sans autres données la variation thermique de l'aimantation spontanée: c'est ainsi qu'on a trouvé pour $\mathrm{Fe}_{2} \mathrm{O}_{3} \mathrm{FeO}$ des résultats en accord avec l'expérience.

Ainsi le ferromagnétisme des ferrites est lié à la présence d'ions magnétiques sur deux catégories de sites, cristallographiquement différents, et à l'existence d'intégrales d'échange négatives: il s'agirait plutôt d'un antiferromagnétisme imparfait que d'un ferromagnétisme et c'est pourquoi il paraîtrait justifié de ranger ces substances dans une catégorie spéciale, celle des terrimagnétiques.

Reçu 21-2-50.

LOUIS NÉEL.

Laboratoire d'Electrostatique et de Physique du Métal, Grenoble. 


\section{LITERATURE}

1) A. Serres, Ann. de Physique, 1932, 17, 53.

2) J. L. Sn o e k, Physica, 1936, 3, 463; New developments in Ferromagnetic Materials Elsevier, Amsterdam, 1947.

3) T. F. W. B a r th et E. Pos n j a k, Z. Krist., 1932, 89, 325.

4) E. J. W. V c r we y et E. L. H e il m a n n, J. chem. Physics, 1947, 15, 174.

5) J. H. Gis olf, Physica, 1949, 15, 677.

6) E. W. Gorter, C. R. Ac. Sc., Paris, 1950, 230, 192; Nature, sous presse.

7) C. Guill a u d, C. R. Ac. Sc., Paris, 1949, 299, 1133.

8) encore inédit.

9) L. N é e 1, Ann. de Physique, 1948, 3, 137; C. R. Ac. Sc., Paris, 1950, 230, 190; L $\mathrm{N}$ é el et P. B rochet, C. R. Ac. Sc., Paris, 1950, 230, 280; L. N é e l, C. R. Ac Sc.. Paris, 1950, 930, 375. 